\title{
A closed-form solution for stress analysis of hollow cylinder structure under non-uniform external load and its engineering application
}

\author{
Xiaoben Liu****, Hong Zhang*, Mengying Xia***, Baodong Wang*, Qian Zheng* and Kai Wu* \\ *College of Mechanical and Transportation Engineering, China University of Petroleum-Beijing 102249, China \\ **Department of Civil and Environmental Engineering, University of Alberta, Edmonton, Canada \\ *Corresponding Author: hzhang@cup.edu.cn
}

\begin{abstract}
Hollow structures are widely used in industry for their high bearing capacity. In some engineering cases, these structures are always subjected to complicated non-uniform external loads. For example, bored (foundation) Piles steel casing and steel casing used for oil production are subjected to non-uniform ground stresses. In this study, a generalized closed-form mathematical solution for stress analysis of hollow cylinder under non-uniform external load was derived. The common non-uniform external load was decomposed by Fourier series under the principle of superposition by theory of elasticity. Analytical solutions for stress results of sine or cosine series external load problems were obtained by the semi-inverse method. A baseline analysis of a steel casing under non-uniform ground stress was presented using the proposed analytical method. And the finite element model by ABAQUS was used to compare and validate the results. A parametric analysis was conducted finally to discuss the effects of non-uniform coefficients on the stress results. Results show that the hollow cylinder structure's anti-collapse capacity will heavily decrease when the non-uniform coefficient increases. This proposed analytical model can be referenced in strength verification of hollow cylinder structures in engineering practice.
\end{abstract}

Keywords: hollow cylinder; non-uniform external pressure; stress analysis; analytical method; engineering application.

\section{O INTRODUCTION}

Hollow cylinder structure is a typical basic structure widely used in mining, water resources and hydropower, nuclear power, chemical, manufacture and petroleum industries, and Foundation (Bored) Piling. Fig. 1 (a) and Fig. 1 (b) show the casing used in petroleum engineering and the shield machine used for tunneling engineering.



(a)

(b)

Fig. 1. Hollow cylinder structures used in industry. 
Under external load, hollow cylinders may experience large stress leading to potential strength failure or large deformation leading potential collapse failure. For hollow cylinder structures that are mainly key bearing components in the industry, stress and deformation of hollow cylinder structure subjected to different loads gained the attention of both researchers and engineers. In this study, an effort was put to develop an analytical stress analysis method of general hollow cylinder structures under environmental loads, especially non-uniform pressure loads, based on theory of elasticity. Non-uniform external loads were decomposed by Fourier series according to superposition principle. Suitable stress functions were adopted in the semi-inverse method in order to calculate the analytical results for hoop, radial, and axial stresses, respectively. Based on the von Mises stress criteria, strength analysis method was established for hollow cylinder structure under non-uniform external load. Finally a baseline analysis was conducted for steel casing under non-uniform ground stress, and effects of non-uniform coefficient on the stress results were discussed. The presented study can be referenced in the strength analysis and safety evaluation of hollow cylinder structures in engineering practice.

\section{LITERATURE REVIEW}

Intensive researches on mechanical analysis of the hollow cylinder structures have been conducted previously. A typical stress analytical solution for the circular cylinder structure is the famous lame solution for axisymmetric loads (Liu D. et al., 1992).

Liang et al. (2008) focused on the analytical stress results for spatially axisymmetric thick-walled cylinder under linear varying pressure in the axial direction. Naumenko et al. (2009) analyzed the hollow cylindrical section's stress state by considering the effects induced by steady-state creep. Boyle (2012) derived the analytical stress results for hollow cylinder based on von Mises yield criteria. It can be summarized that they are all focusing on circumferential uniform external load.

However, the hollow cylinder structures are always subjected to unsymmetrical external loads in actual engineering, for example, the shaft lining in mining, circular tunnel in civil engineering projects, and the oil steel casing in petroleum engineering. The non-uniform loads are mainly caused by the anisotropy of the rocks and strata around these structures, the rock stratum movement, the initial geo stress field, etc. Green (1948) analyzed the stress results for the infinite plate with a circular hole, which can be considered as the pioneer work of stress analysis for the structure subjected to unsymmetrical external loads. Sternberg et al. (1949) also conducted some numerical analysis for similar problems with modified Ritz method. Zheng et al. (1998) studied the strength of the circular casing under non-uniform load induced by ground stress based on the stress function method. Wu Q. et al. (2011) proposed an analytical stress calculation model for a typical type of non-uniform pressure and solved by complex function method. Liu et al. $(2006,2009)$ also derived stress analytical results for the cylinder under the external load with an exponential type function distribution. Zhou Z. (2007) calculated the stress field and displacement field of the ideal casing under uniform and non-uniform external pressure, respectively, based on ANSYS. Yang et al. (2011) proposed an analytical model based on the thick cylinder plane stress theory of the stress calculation for the casing subjected to the internal and external pressure, temperature, and axial force. Wang Y.F. (2009) established the finite element model of the casing with abrasion defects and considered the coupling effects of thermal stress and external load. It is noted that the external pressures on the casing in papers of Yang (2011) and Wang Y.F. (2009) are both considered as uniform. Kampf-Sherf O. et al. (2012) discussed the effect of stress concentration caused by corrosion cavity on the casing strength and established the mathematical model that can obtain the stress concentration factor caused by corrosion cavity based on the knowledge of plastic-elasticity. Deng K.H. et al. (2016) put forward the conception of advance coefficient of plastic collapse of casing under non-uniform load based on the elastic theory and the new ISO collapse model, and established the new equations to compute casing collapse resistance under non-uniform load. Irawan S. et al. (2015) estimated casing burst strength after wear based on the finite element analysis and compared the different theoretical bursts pressure with the simulation results along with effect of different wear shaper on L-80 casing material. Dong S.M. et al. (2015) established the elastic mechanical model of the combination composed of 
casing-cement ring and the surrounding rock and discussed casing stress distribution in different injection-production cycle, combining with the thermal recovery routine parameters of a certain oilfield.

Nevertheless, the existing methods are all just suitable for a special kind of external load distribution. Less attention is paid to a more generalized method. To solve this scientific problem, a closed-form solution of the hollow cylinder stress analysis under general non-uniform external load was proposed in this study. Any non-uniform type external load is treated as a combination of uniform load, cosine series load, and sine series load, which makes a wider applicability of the presented method.

\section{MATHEMATICAL SOLUTION FOR HOLLOW CYLINDER UNDER NON-UNIFORM EXTERNAL PRESSURE}

\subsection{Mechanical Model}

The hollow cylinder will encounter various kinds of load in actual working conditions, and the non-uniform external load is a typical form, which occurs in a variety of situations, e.g., casing under non-uniform ground stress. When the hollow circular is subjected to uniform internal pressure and non-uniform external pressure, the condition can be treated as plane strain case when neglecting the longitudinal strain, as shown in Fig. 2.

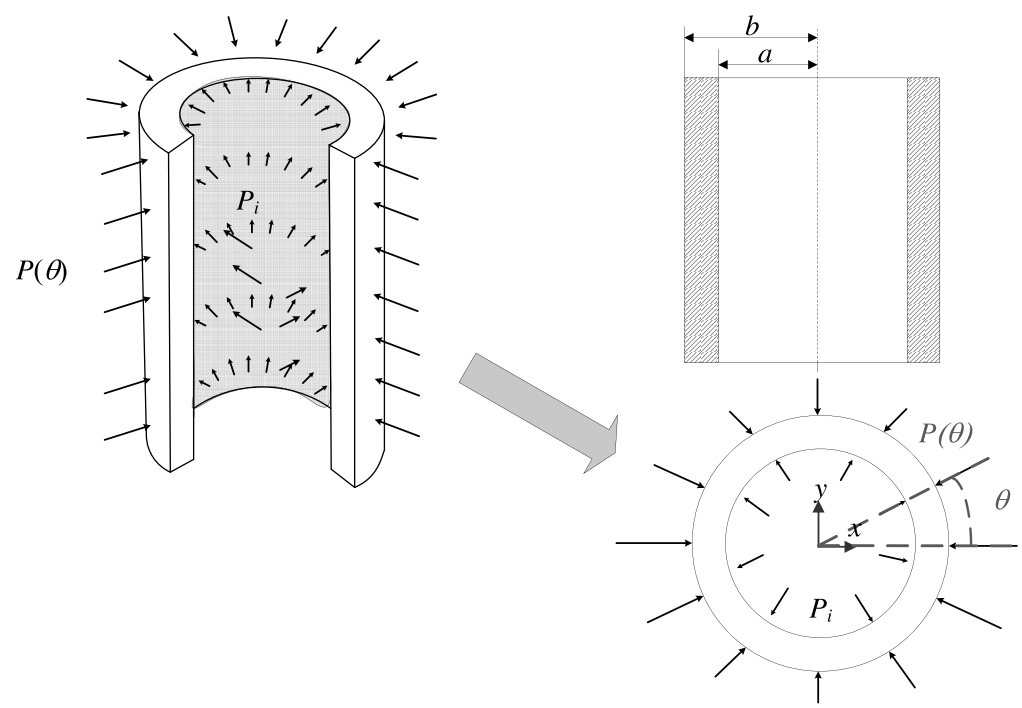

Fig. 2. Sketch of hollow cylinder subjected to internal and non-uniform external pressure.

The research considered the elastic problem. Based on this assumption, the non-uniform external pressure applied on the hollow cylinder can be decomposed by Fourier series with the principle of superposition. A Fourier series is an expansion of a periodic function in terms of an infinite sum of sines and cosines. Fourier series make use of the orthogonality relationships of the sine and cosine functions. The computation and study of Fourier series are extremely useful as a way to break up an arbitrary periodic function into a set of simple terms that can be plugged in, solved individually, and then recombined to obtain the solution to the original problem or an approximation to it to whatever accuracy is desired or practical. Thus, for this study, the non-uniform load can be also expressed as follows.

$$
P(\theta)=\frac{1}{2} a_{0}+\sum_{n=1}^{\infty}\left(a_{n} \cos n \theta+b_{n} \sin n \theta\right)
$$


where

$$
\begin{aligned}
& a_{0}=2 P_{0}=\frac{1}{\pi} \int_{-\pi}^{\pi} P(\theta) d \theta \\
& a_{n}=\frac{1}{\pi} \int_{-\pi}^{\pi} P(\theta) \cos n \theta d \theta \quad(n=1,2 \cdots) \\
& b_{n}=\frac{1}{\pi} \int_{-\pi}^{\pi} P(\theta) \sin n \theta d \theta \quad(n=1,2, \cdots)
\end{aligned}
$$

where $P(\theta)$ is non-uniform external load, $a_{0}$ is constant in the Fourier series expansion, $\mathrm{P}_{0}$ is the constant component of the external , $a_{n}$ and $b_{n}$ are expansion coefficients in the Fourier series expansion, $n$ is the number of items needed for decomposition of the un-uniform external loads, and $\theta$ is the angle between the normal direction of the external load and the $x$-axis in the coordinate illustrated in Fig 2.

Therefore, the internal and external pressure load can be decomposed into three kinds of loads: the uniform internal and external pressure, cosine series external pressure, and sine series external pressure. The total stress of hollow cylinder under non-uniform external pressure can be calculated by the superposition results of these three loads.

\subsection{Solutions for uniform internal and external pressure problem}

The uniform internal and external pressure problem of hollow cylinder are a classic problem in elastic mechanics, in which the hollow cylinder is free from shear stress on the edge (Lu M. 2001). The boundary conditions for this problem are as follows:

$$
\left\{\begin{array}{lll}
\sigma_{r}=-P_{0}, & \tau_{r \theta}=0 & r=b \\
\sigma_{r}=-P, & \tau_{r \theta}=0 & r=a
\end{array}\right.
$$

where $a$ is the inner radius, $b$ is the outer radius, $P$ is the uniform internal pressure, $P_{0}$ is the component of the uniform external pressure, $\sigma_{r}$ is the radial stress, and $\tau_{r \theta}$ is the shear stress.

The analytical solution can be directly obtained by Lame formula and Poisson's ratio (Xu Z. 1990); namely,

$$
\begin{aligned}
& \sigma_{r p}=\frac{a^{2} b^{2}\left(P-P_{0}\right)}{\left(a^{2}-b^{2}\right) r^{2}}+\frac{\left(a^{2} P-b^{2} P_{0}\right)}{b^{2}-a^{2}} \\
& \sigma_{\theta p}=\frac{a^{2} P-b^{2} P_{0}}{b^{2}-a^{2}}-\frac{a^{2} b^{2}\left(P-P_{0}\right)}{\left(a^{2}-b^{2}\right) r^{2}} \\
& \tau_{p}=0 \\
& \sigma_{z p}=v\left(\sigma_{r p}+\sigma_{\theta p}\right)
\end{aligned}
$$

where $\sigma_{r p}$ is the radial stress for uniform external load, $\sigma_{\theta p}$ is the hoop stress for uniform external load, $\sigma_{z p}$ is the axial stress for uniform external load, $\tau_{p}$ is the shear stress for uniform external load, and $v$ is Poisson's ratio.

\subsection{Solutions for cosine series external pressure problem}

In this section, the closed-form solution for stress of hollow cylinder subjected to cosine series external load was proposed. When the cosine series load was applied, according to the boundary conditions, the stress function can be assumed

$$
\phi=f(r) \cos n \theta
$$


Taking this stress function into the bi-harmonic equation, the differential equation of stress function can be obtained as

$$
\nabla^{4} \phi=\left(\frac{\partial^{2}}{\partial r^{2}}+\frac{1}{r} \frac{\partial}{\partial r}+\frac{1}{r^{2}} \frac{\partial^{2}}{\partial \theta^{2}}\right)\left(\frac{\partial^{2}}{\partial r^{2}}+\frac{1}{r} \frac{\partial}{\partial r}+\frac{1}{r^{2}} \frac{\partial^{2}}{\partial \theta^{2}}\right) \phi=0
$$

where $\phi$ is stress function.

The general solution of stress function can be obtained as follows:

$$
\phi= \begin{cases}\left(A_{1} r^{3}+B_{1} r^{-1}+C_{1} r+D_{1} r \ln r\right) \cos \theta & n=1 \\ \left(A r^{-n}+B r^{2-n}+C r^{2+n}+D r^{n}\right) \cos n \theta & n \geq 2\end{cases}
$$

It should be noted that the result for $n=1$ is applicable for wind load, which is not necessary to be considered in this paper. Thereby, taking the stress function for $n \geq 2$ into Equation (8),

$$
\begin{aligned}
& \sigma_{r}=\frac{1}{r} \frac{d \phi}{d r}+\frac{1}{r^{2}} \frac{\partial^{2} \phi}{\partial \theta^{2}} \\
& \sigma_{\theta}=\frac{\partial^{2} \phi}{\partial r^{2}} \\
& \tau_{r \theta}=-\frac{\partial}{\partial r}\left(\frac{1}{r} \frac{\partial \phi}{\partial \theta}\right) \\
& \sigma_{z}=v\left(\sigma_{r}+\sigma_{\theta}\right)
\end{aligned}
$$

the stress components can be obtained as

$$
\begin{aligned}
\sigma_{n r \cos } & =-r^{-2-n}\left\{A n(1+n)+B\left(-2+n+n^{2}\right) r^{2}+r^{2 n}\left[D(-1+n) n+C\left(-2-n+n^{2}\right) r^{2}\right]\right\} \cos (n \theta) \\
\sigma_{n \theta \cos } & =\left[-A(-1-n) n r^{-2-n}+D(-1+n) n r^{-2+n}+B(1-n)(2-n) r^{-n}+C(1+n)(2+n) r^{n}\right] \cos (n \theta) \\
\tau_{n r \theta \cos } & =n r^{-2-n}\left\{-A(1+n)-B(-1+n) r^{2}+r^{2 n}\left[D(-1+n)+C(1+n) r^{2}\right]\right\} \sin (n \theta) \\
\sigma_{n z \cos } & =v\left(\sigma_{n r \cos }+\sigma_{n \theta \cos }\right)
\end{aligned}
$$

where $n$ is the number of the series, $A, B, C, D$ are coefficients, $\sigma_{n r \text { cos }}$ is the radial stress for cosines external load, $\sigma_{n \theta \cos }$ is the hoop stress for cosines external load, $\sigma_{n z \cos }$ is the axial stress for cosines external load, $\tau_{n r \theta \cos }$ is the shear stress for cosines external load, and $n$ is the number of items needed for decomposition of the un-uniform external loads

Then, substituting the boundary conditions below into Equations (9),

$$
\left\{\begin{array}{lrl}
\sigma_{r}=-a_{n} \cos n \theta ; & \tau_{r \theta}=0 & r=b \\
\sigma_{r}=0 ; & \tau_{r \theta}=0 & r=a
\end{array}\right.
$$

values of coefficients, $A$ to $D$, can be determined as follows. 


$$
\begin{aligned}
& A=-\frac{a_{n} a^{2+2 n} b^{2+n}\left[a^{2 n} b^{2}+a^{2} b^{2 n} n-b^{2+2 n}(1+n)\right]}{2(1+n)\left[a^{2+4 n} b^{2}+a^{2} b^{2+4 n}-a^{4+2 n} b^{2 n} n^{2}-a^{2 n} b^{4+2 n} n^{2}+2 a^{2+2 n} b^{2+2 n}\left(-1+n^{2}\right)\right]} \\
& B=-\frac{a_{n} a^{2 n} b^{2+n}\left(-a^{2+2 n}+a^{2} b^{2 n}-a^{2} b^{2 n} n+b^{2+2 n} n\right)}{2(-1+n)\left(a^{2+4 n} b^{2}-2 a^{2+2 n} b^{2+2 n}+a^{2} b^{2+4 n}-a^{4+2 n} b^{2 n} n^{2}+2 a^{2+2 n} b^{2+2 n} n^{2}-a^{2 n} b^{4+2 n} n^{2}\right)} \\
& C=-\frac{a_{n} b^{2+n}\left(-a^{2+2 n}+a^{2} b^{2 n}-a^{2+2 n} n+a^{2 n} b^{2} n\right)}{2(1+n)\left(a^{2+4 n} b^{2}-2 a^{2+2 n} b^{2+2 n}+a^{2} b^{2+4 n}-a^{4+2 n} b^{2 n} n^{2}+2 a^{2+2 n} b^{2+2 n} n^{2}-a^{2 n} b^{4+2 n} n^{2}\right)} \\
& D=\frac{a_{n} a^{2} b^{2+n}\left(-a^{2 n} b^{2}+b^{2+2 n}-a^{2+2 n} n+a^{2 n} b^{2} n\right)}{2(-1+n)\left(a^{2+4 n} b^{2}-2 a^{2+2 n} b^{2+2 n}+a^{2} b^{2+4 n}-a^{4+2 n} b^{2 n} n^{2}+2 a^{2+2 n} b^{2+2 n} n^{2}-a^{2 n} b^{4+2 n} n^{2}\right)}
\end{aligned}
$$

Thus, the stress of hollow cylinder under cosine series external pressure can be derived after substituting coefficients shown in Equation (11) into Equation (9).

\subsection{Solutions for sine series external pressure problem}

Similarly, the stress formula of hollow cylinder under sine series external pressure is assumed as follows.

$$
\phi=f(r) \sin n \theta
$$

Taking stress function into bi-harmonic Equation (6), the general solution of stress function considered in this study can be obtained as follows:

$$
\phi=\left(A_{2} r^{-n}+B_{2} r^{2-n}+C_{2} r^{2+n}+D_{2} r^{n}\right) \sin n \theta
$$

Then the stress components can be also derived:

$$
\begin{aligned}
\sigma_{n r \sin } & =-r^{-2-n}\left\{A_{2} n(1+n)+B_{2}\left(-2+n+n^{2}\right) r^{2}+r^{2 n}\left[D_{2}(-1+n) n+C_{2}\left(-2-n+n^{2}\right) r^{2}\right]\right\} \sin (n \theta) \\
\sigma_{n \theta \sin } & =\left[-A_{2}(-1-n) n r^{-2-n}+D_{2}(-1+n) n r^{-2+n}+B_{2}(1-n)(2-n) r^{-n}+C_{2}(1+n)(2+n) r^{n}\right] \sin (n \theta) \\
\tau_{n r \theta \sin } & =n r^{-2-n}\left\{A_{2}(1+n)+B_{2}(-1+n) r^{2}-r^{2 n}\left[D_{2}(-1+n)+C_{2}(1+n) r^{2}\right]\right\} \cos (n \theta) \\
\sigma_{n z \sin } & =v\left(\sigma_{n r \sin }+\sigma_{n \theta \sin }\right)
\end{aligned}
$$

According to the boundary conditions,

$$
\left\{\begin{array}{lll}
\sigma_{r}=-b_{n} \sin n \theta & \tau_{r \theta}=0 & r=b \\
\sigma_{r}=0 & \tau_{r \theta}=0 & r=a
\end{array}\right.
$$

Values of coefficients, $A_{2}$ to $D_{2}$, can also be determined as follows.

$$
\begin{aligned}
& A_{2}=-\frac{b_{n} a^{2+2 n} b^{2+n}\left[a^{2 n} b^{2}+a^{2} b^{2 n} n-b^{2+2 n}(1+n)\right]}{2(1+n)\left(a^{2+4 n} b^{2}+a^{2} b^{2+4 n}-a^{4+2 n} b^{2 n} n^{2}-a^{2 n} b^{4+2 n} n^{2}+2 a^{2+2 n} b^{2+2 n}\left(-1+n^{2}\right)\right)} \\
& B_{2}=-\frac{b_{n} a^{2 n} b^{2+n}\left(-a^{2+2 n}+a^{2} b^{2 n}-a^{2} b^{2 n} n+b^{2+2 n} n\right)}{2(-1+n)\left(a^{2+4 n} b^{2}-2 a^{2+2 n} b^{2+2 n}+a^{2} b^{2+4 n}-a^{4+2 n} b^{2 n} n^{2}+2 a^{2+2 n} b^{2+2 n} n^{2}-a^{2 n} b^{4+2 n} n^{2}\right)} \\
& C_{2}=-\frac{b_{n} b^{2+n}\left(-a^{2+2 n}+a^{2} b^{2 n}-a^{2+2 n} n+a^{2 n} b^{2} n\right)}{2(1+n)\left(a^{2+4 n} b^{2}-2 a^{2+2 n} b^{2+2 n}+a^{2} b^{2+4 n}-a^{4+2 n} b^{2 n} n^{2}+2 a^{2+2 n} b^{2+2 n} n^{2}-a^{2 n} b^{4+2 n} n^{2}\right)} \\
& D_{2}=\frac{b_{n} a^{2} b^{2+n}\left(-a^{2 n} b^{2}+b^{2+2 n}-a^{2+2 n} n+a^{2 n} b^{2} n\right)}{2(-1+n)\left(a^{2+4 n} b^{2}-2 a^{2+2 n} b^{2+2 n}+a^{2} b^{2+4 n}-a^{4+2 n} b^{2 n} n^{2}+2 a^{2+2 n} b^{2+2 n} n^{2}-a^{2 n} b^{4+2 n} n^{2}\right)}
\end{aligned}
$$


where $n$ is the number of the series, $A_{2}, B_{2}, C_{2}, D_{2}$ are coefficients, $\sigma_{n r \sin }$ is the radial stress for sine external load, $\sigma_{n \theta \sin }$ is the hoop stress for sine external load, $\sigma_{n z \sin }$ is the axial stress for sine external load, $\tau_{n r \theta \sin }$ is the shear stress for sine external load, and $n$ is the number of items needed for decomposition of the un-uniform external loads.

The stress for sine series external pressure will be obtained after substituting coefficients in Equation (16) into Equation (14).

\subsection{Stress for general non-uniform external pressure}

Superposing results of Equations (4), (9), and (14), stress components for hollow cylinder structure under a general non-uniform external pressure can be derived as follows.

$$
\left\{\begin{array}{l}
\sigma_{r}=\sigma_{r p}+\sum_{n=2}^{\infty} a_{n} \sigma_{n r \cos }+\sum_{n=2}^{\infty} b_{n} \sigma_{n r \sin } \\
\sigma_{\theta}=\sigma_{\theta p}+\sum_{n=2}^{\infty} a_{n} \sigma_{n \theta \cos }+\sum_{n=2}^{\infty} b_{n} \sigma_{n \theta \sin } \\
\sigma_{z}=\sigma_{z p}+\sum_{n=2}^{\infty} a_{n} \sigma_{n z \cos }+\sum_{n=2}^{\infty} b_{n} \sigma_{n z \sin } \\
\tau_{r \theta}=\sum_{n=2}^{\infty} a_{n} \tau_{n z \cos }+\sum_{n=2}^{\infty} b_{n} \tau_{n z \sin }
\end{array}\right.
$$

\section{6 von Mises yield criterion}

Elastic buckling and plastic collapse failure will occur to the hollow cylinder structure under non-uniform external pressure. Related researches show that plastic buckling occurs when the diameter-thickness ratio is larger than 32 (Li J. 2005). As the ratio for most of the existing steel casings is not more than 32, only the plastic collapse failure can possibly occur for these casings. For typical ductile steel materials, the von Mises yield criterion is suitable for yield recognition. Based on this criterion, the structure can be considered as yield if the peak second deviatoric stress invariant in the structure reaches a critical value, as shown in Equation (18).

$$
\sigma_{\text {Mises }}=\sqrt{3 J_{2}}=\sqrt{\frac{1}{2}\left[\left(\sigma_{11}-\sigma_{22}\right)^{2}+\left(\sigma_{11}-\sigma_{33}\right)^{2}+\left(\sigma_{22}-\sigma_{33}\right)^{2}+6\left(\tau_{12}^{2}+\tau_{13}^{2}+\tau_{23}^{2}\right)\right]} \geq \sigma_{s}
$$

where $J_{2}$ the second deviatoric stress invariant, $\sigma_{\text {Mises }}$ is von Mises stress, $\sigma_{i j}$ are the Cauchy stress tensor components, which can derived by Equation (16), and $\sigma_{s}$ is the yield strength, which is $770 \mathrm{MPa}$ for P110 casing steel.

\subsection{Solution algorithm}

The solution algorithm of the presented method for hollow cylinder structure under non-uniform external load was illustrated in Fig. 3. 

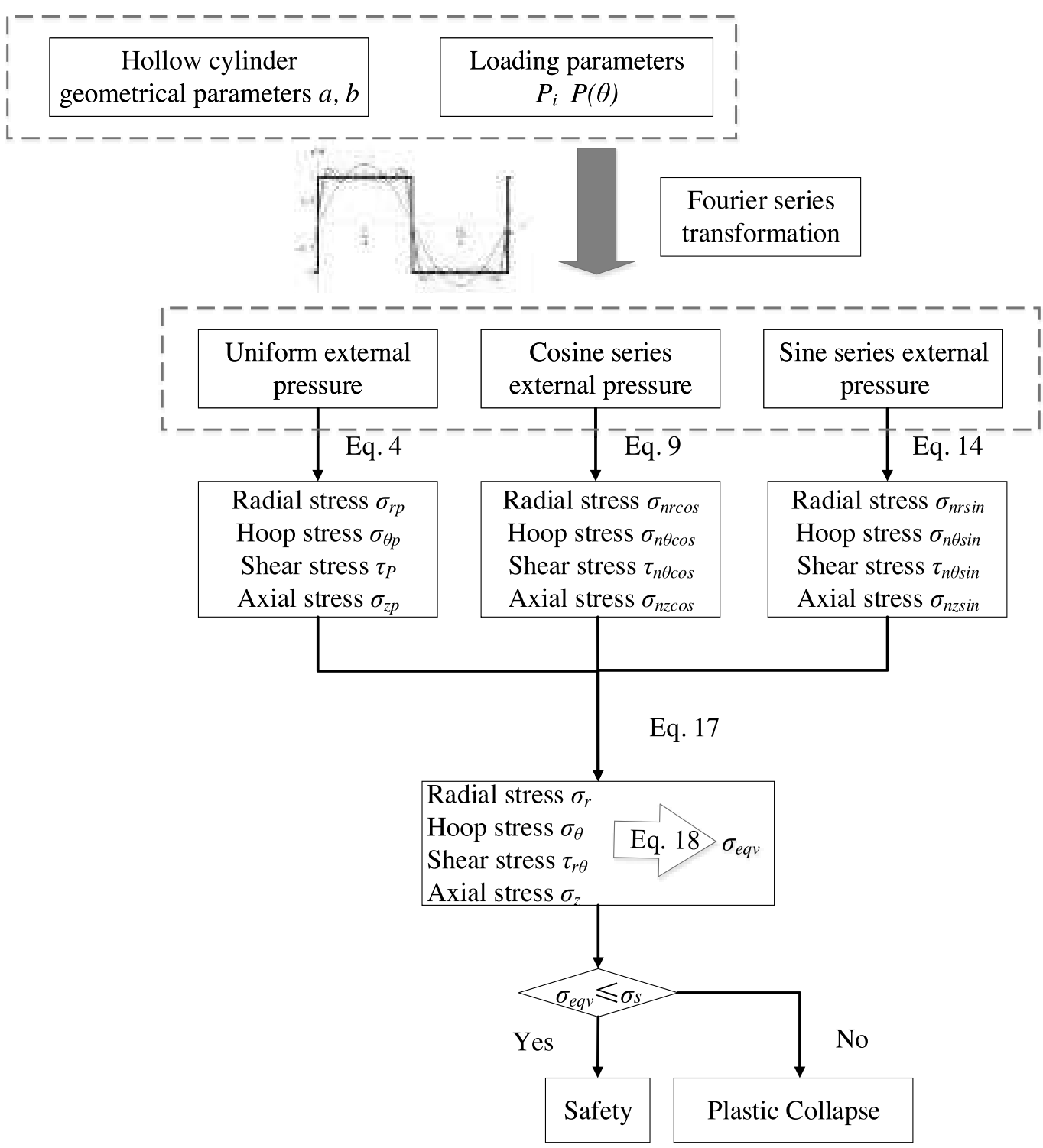

Fig. 3. Flow chart for the proposed mathematical method.

\section{MODELLING HOLLOW CYLINDER SECTIONS USING FINITE ELEMENT METHOD}

The finite element (FE) model used by Li J. (2005) was also adopted here to validate the accuracy of the propose model. General FE software package ABAQUS was utilized to establish the numerical model. In the FE model, four-node plane strain element CPE4 was selected to model the casing. The element number was set to be 400 after convergence and sensible analysis. An elastic material was used, as the mechanical problem is based on elastic assumption. A nonlinear static analysis was performed to solve this problem, with internal pressure and non-uniform external pressure being applied on the inner surface and outer surface, respectively. 


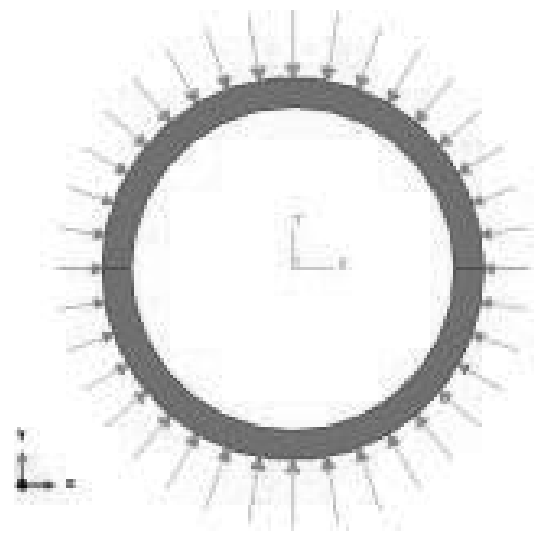

(a) Non uniform external load on hollow cylinder

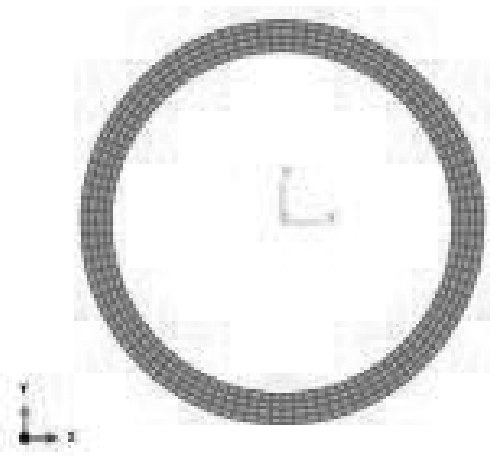

(b) Mesh details by plane strain elements

Fig. 4. Finite element model for hollow cylinder under non-uniform external load.

\section{CASE STUDY FOR CASING UNDER NON-UNIFORM GROUND STRESS}

\subsection{Baseline analysis for validation of the proposed analytical model}

When a casing is subjected to non-uniform ground stress, it can be treated as a typical hollow cylinder under non-uniform external pressure. In this section, the effect of non-uniform external pressure on stress distribution in the hollow cylinder was analyzed with actual data of a casing in engineering. The distribution of creep external load of mudstone around the casing can be approximately expressed by a cosine function (Li J. 2005).

$$
\sigma_{n}=S_{1}+S_{2} \cos 2 \theta
$$

where $\sigma_{n}$ is radial external ground creep pressure, MPa; $\theta$ is the maximum angle between ground stress and $\sigma_{n},{ }^{\circ}$; and $S_{1}, S_{2}$ are conversion loads of ground stress, $S_{l}=1 / 2\left(\sigma_{H^{+}} \sigma_{h}\right), S_{2}=1 / 2\left(\sigma_{H^{-}}-\sigma_{h}\right)$, in which $\sigma_{H}$ and $\sigma_{h}$ are radial external creep pressure of casing in the direction of maximum and minimum ground stress, MPa.

According to Li J.'s (2005) investigation, some related parameters were derived as follows. When the well depth is $2000 \mathrm{~m}$, the creep load of casing under uniform ground stress can be considered as $\sigma_{H}=\sigma_{h}=29.4 \mathrm{MPa}$. To simulate different conditions of original ground stress, five non-uniform coefficients $\delta=\sigma_{H /} \sigma_{h}$ were selected, i.e., $\delta=1.1,1.2,1.3$, 1.4, and 1.5. The type of P110 casing pipe with the outer diameter of $177.8 \mathrm{~mm}$ and wall thickness of $13.72 \mathrm{~mm}$ was analyzed. Its elastic modulus is $210 \mathrm{GPa}$, the Possion's ratio is 0.26 , and the yield strength is $770 \mathrm{MPa}$.

von Mises stress contours for $\delta=1.5$ derived by finite element method illustrated in Section 3 and the proposed analytical method were both illustrated in Fig. 5. The calculating code by MATLAB of the presented mathematical method was listed in the Appendix. The figure shows that both stress values and stress distribution are almost the same for the two methods, which proves that the proposed close-form analytical solution is accurate. 


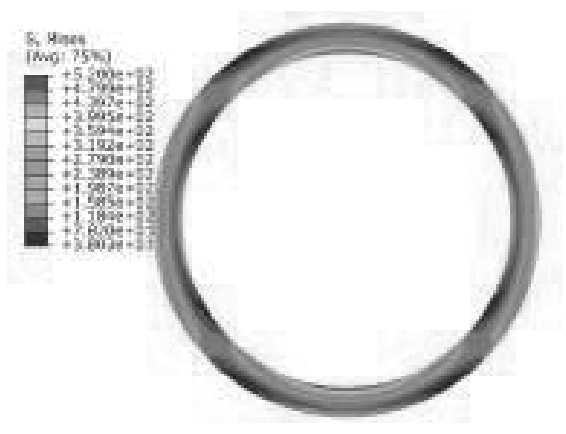

a) Stress contour of FE model results (MPa)

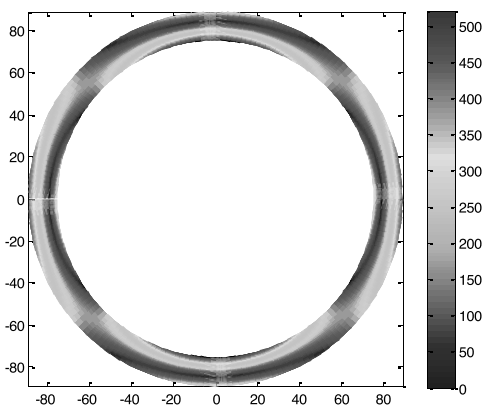

b) Stress contour of proposed model (MPa)

Fig. 5. Mises stress distribution when $\delta=1.5$ derived from ABAQUS and the proposed method.

\subsection{Effects of the non-uniform coefficients on stress results}

Parametric analysis was further conducted in this section to analyze the effects induced by the non-uniform coefficient. Table 1 shows the relationships of the maximum von Mises stress in the cylinder with the non-uniform coefficient $\delta$, for both numerical and analytical results. It can be obtained that the two series of results are almost the same with each other. The maximum relative error between them for the five cases considered is only $1.16 \%$, which is negligible in engineering application.

Table 1. Comparison of the maximum equivalent Mises stress in casing with various values of $\delta$.

\begin{tabular}{|c|c|c|c|c|c|}
\hline Non-uniform Coefficient & 1.1 & 1.2 & 1.3 & 1.4 & 1.5 \\
\hline Mathematical solution & 275.57 & 352.32 & 417.26 & 472.92 & 521.16 \\
\hline Finite element results & 272.4 & 350.2 & 415.6 & 471.8 & 520 \\
\hline Relative error & $1.16 \%$ & $0.61 \%$ & $0.40 \%$ & $0.24 \%$ & $0.22 \%$ \\
\hline
\end{tabular}

And with increase of $\delta$, the maximum von Mises stress increases obviously, which makes the anti-collapse capacity of casing weakened. The von Mises distribution in the outer surface and the von Mises contours of cylinder under different non-uniform external pressure were also elaborated in Fig. 6 and Fig. 7, respectively, to analyze the structure performance in detail.

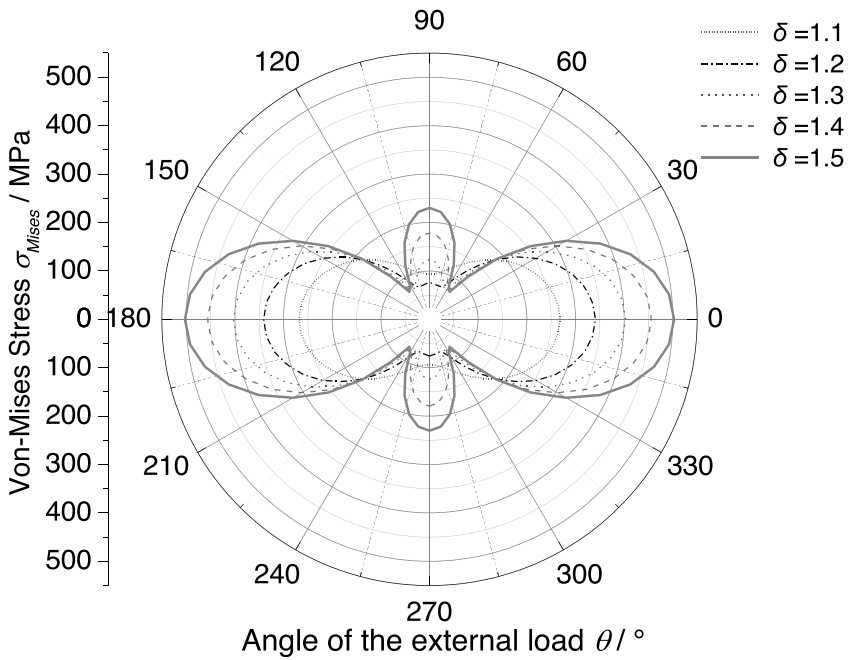

Fig. 6. von Mises stress distribution in the out surface with different non-uniform coefficients. 
From the contours, with increase of $\delta$, large stress starts to concentrate in the inner surface at the top and inverse and the outer surface in the lateral from plane view. And the peak von Mises stress in the inner surface is larger than that of the outer surface. The von Mises stress distribution of outer surface in the circumferential direction in Fig 7 shows the detailed stress values under different non-uniform coefficients. They are smaller than the maximum values shown in Fig. 5.

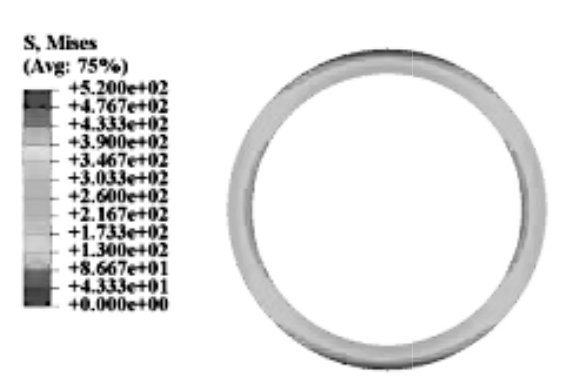

a) Stress contour of FE model results (MPa)


b) Stress contour of proposed model (MPa)

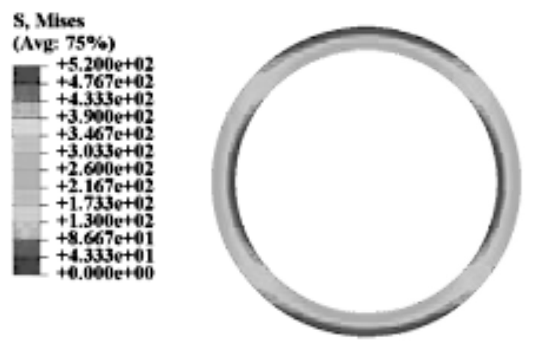

c) Stress contour of FE model results (MPa)
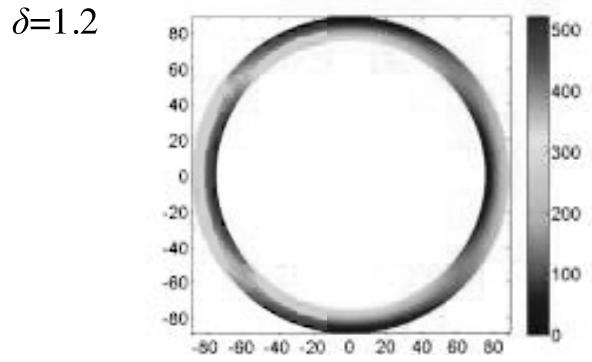

d) Stress contour of proposed model (MPa)
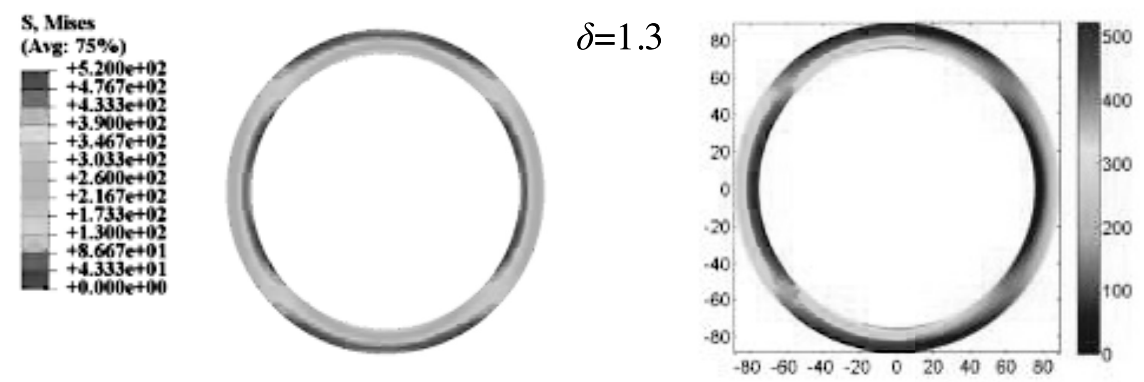

e) Stress contour of FE model results (MPa)

f) Stress contour of proposed model (MPa)
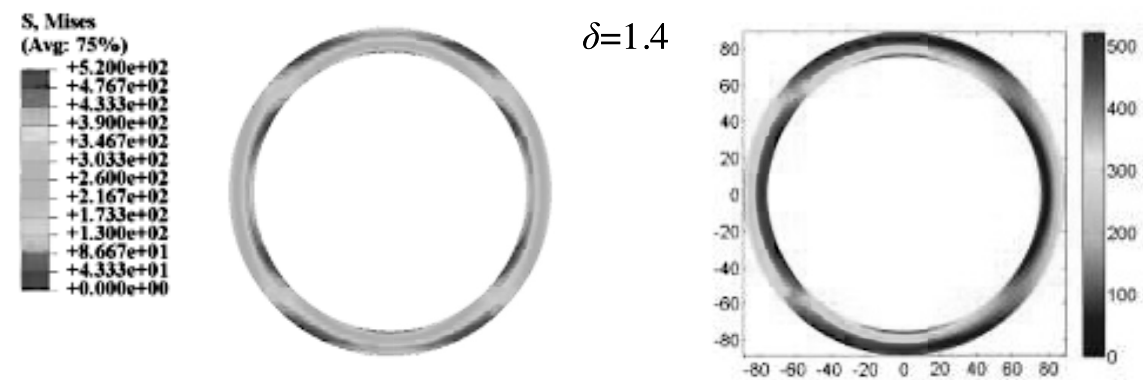

g) Stress contour of FE model results (MPa)

h) Stress contour of proposed model (MPa)

Fig. 7. Comparison results of the presented method and FE model results with various non-uniform coefficients. 
von Mises stresses were mainly induced by the principle stresses. For the shear stress in the presented mode that is relatively small, only the hoop, radial, and axial stress components were discussed here to find the main reason for the increase of von Mises stress induced by non-uniform coefficient. Fig. 8, 9, and 10 illustrate the radial, hoop, and axial stress distribution of the out surface of casing, respectively. It can be derived from these three figures that the radial stress has the smallest value among them. What is more, the non-uniformity of the radial stress is also the most inconspicuous one. The hoop stress and axial stress have similar distribution, while the axial stress value is about onethird of the hoop stress, which is induced by Poisson's ratio. Thus, the von Mises stress was mainly caused by the hoop stress, which explains that their stress distribution patterns are both highly non-uniform.



Fig. 8. Radial stress distribution in the outer surface with different values of $\delta$.

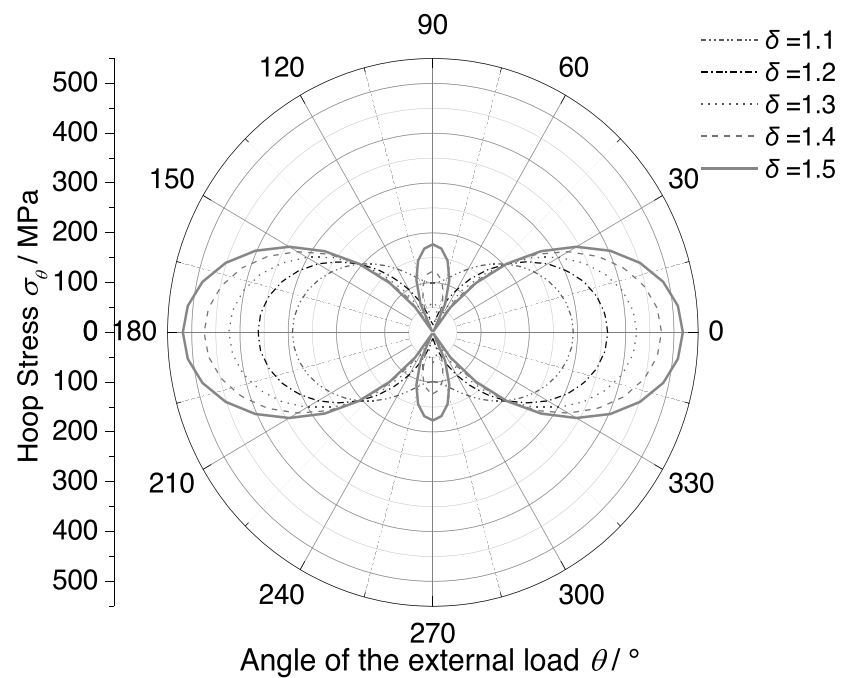

Fig. 9. Hoop stress distribution in the outer surface with different values of $\delta$. 


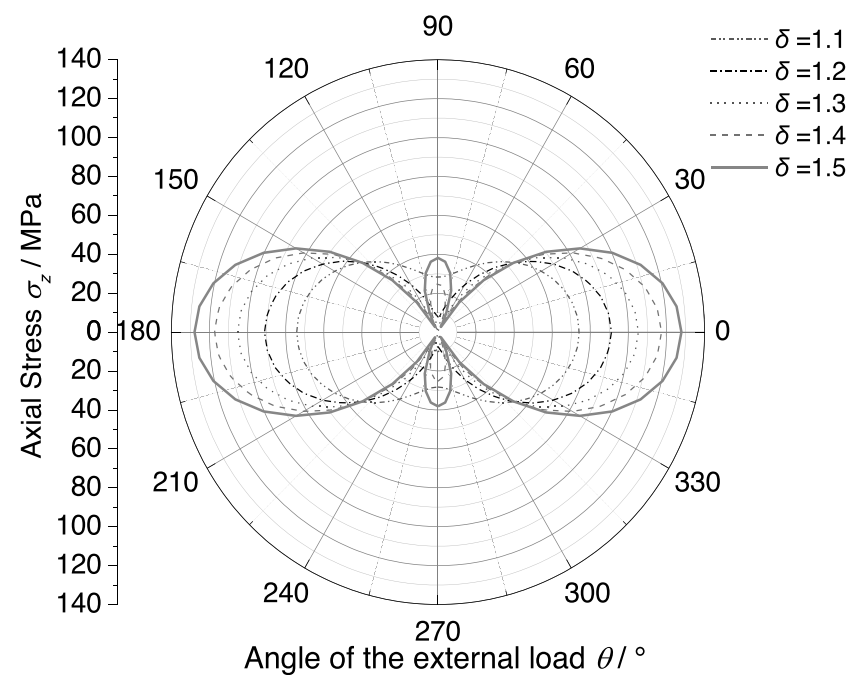

Fig. 10. Axial stress distribution in the outer surface with different values of $\delta$.

\section{CONCLUSIONS}

Hollow cylinder type structures are widely adopted in industry. In actual engineering cases, the external load of these structures can be complicated. In the presented study, an explicit mathematical solution was derived for hollow cylinder under general non-uniform external load based on theory of elastic. Conclusions are drawn as follows:

1. Based on the principle of superposition, common non-uniformed external load can be decomposed into sine or cosine series type external load problem, whose stress results can be solved using the semi-inverse method.

2. Accuracy of the proposed closed-form solution was validated by comparing with finite element model results solved by recognized general FEM software ABAQUS. For the steel casing problem considered in this study, the maximum error of the proposed mathematical method is only $1.16 \%$.

3. The von Mises stress in the hollow cylinder under non-uniform load was mainly induced by the hoop stress. With the increase of the non-uniform coefficient, the hoop stress increases obviously leading to the increasing of von Mises stress, which finally reduces the anti-collapse capacity of the structure.

\section{ACKNOWLEDGMENT}

The work was financially supported by Science Foundation of China University of Petroleum, Beijing (Grants No. 2462018YJRC019, No. 2462018QZDX03) and China National Key Research and Development Project under Grant No. 2016YFC0802105.

\section{REFERENCES}

Boyle, J.T. 2012. The creep behavior of simple structures with a stress range-dependent constitutive model. Archive of Applied Mechan. 82(4) pp: 495-514.

Deng K. Lin Y. Liu W. et al. 2016. Equations to Calculate Casing Collapse Strength under Nonuniform Load Based on New ISO Model. Journal of Pressure Vessel Technology, 138(5)

Dong S. Yang J. 2015. Elastoplastic Analysis of Casing Stress in Thermal Recovery. International Power, Electronics and Materials Engineering Conference.

Green A.E. 1948. Three-dimensional stress systems in isotropic plates I. Philos. Transa. of the Royal Society of London. Series A, Mathem. and Physical Sciences, 240(825) pp: 561-597. 
Irawan S. Bharadwaj A.M. Temesgen B. et al. 2015. Effect of wear on the burst strength of 1-80 steel casing, 100(1):012027.

Kampf-Sherf O. Zlotogorski Z. Gillboa A. et al. 2012. Analysis on the influence of corrosion on casing strength in acidic gas field development. Oil Drilling \& Production Technology, 34(4):114-118.

Liu D.C., Zhao Y. 1992. Stability analysis of cylindrical shells under external pressures by half analytical finite element method. Engine. Mechan. 9(1) pp: 104-114.

Li J. 2005. Real-time monitoring and effects of complicated ground stress on casing damage $\mathrm{Ph}$. $\mathrm{D}$ dissertation of China University of Petroleum-Beijing, China. pp: 98-102.

Liu W. 2006. Mathematical model and analytical solution for cylinder subject to uneven pressures. Chinese Journal of Mechan. Engine. 19(4) pp: 574-578.

Liu W. Shan R. 2009. Mathematic model and analytic solution for a cylinder subject to exponential function. Chinese Journal of Mechan. Engine. 22(4) pp: 587-593.

Liang Y.P. 2008. Analytical solution for spatially axisymmetric problem of thick-walled cylinder subjected to different linearly varying pressures along the axis and uniform pressures at two ends. Science in China, 51(1) pp: 98-104.

Lu M., Luo X. 2001. Bases of Elastic Mechanics, Beijing, Tsinghua University Press. pp: 193-200.

Naumenko K., Altenbach H., and Gorash Y. 2009. Creep analysis with a stress range dependent constitutive model. Archive of Applied Mechan. 79(6) pp: 619-630.

Sternberg E., Sadowsky M.A. 1949. Three dimensional solution for the stress concentration around a circular hole in a plate of arbitrary thickness [J]. Journal of Applied Mechan. 16(1) pp: 27-38.

Wang Y.F. 2009. Research on Collapse Strength of Casing in the Oil and Water Well [D]. Xi' an Shiyou University.

Wu, Q.L., Lu, A.Z. 2011. Stress analytical solution for plane problem of a thick-walled cylinder subjected to a type of non-uniform distributed pressures. Engine. Mechan. 6(1), 4-10.

Xu Z. 1990. Elastic-plastic mechanics, Beijing, Higher Education Press.

Yang X.J. Li Z.F. 2011. Mechanical Mechanism of Casing Failure of Thermal Recovery Wells. Journal of Chengde Petroleum College.

Zheng J. Zhang Y.Q., Wang W.J. 1998. Calculation of casing strength under non-uniform loading. Acta Petrolei. Sinica. 19 pp: 119-123.

Zhou Z. 2007. Research on tubular collapse mechanism by computer [D]. Hebei University of Technology. 


\section{Appendix}

Calculation program of the mathematical model coded by MATLAB for the case study described in in Section 4.1:

clc;clear;close all

$\%$ Parameter units

$\%$ Length unit: mm

\% Pressure unit: $\mathrm{MPa}$

$\%$ Internal pressure

$\mathrm{P}=0$;

$\%$ The maximum ground stress

Sig1=29.4;

$\%$ The minimum ground stress

Sig2=29.4/1.5;

$\%$ The outer radius

$b=177.8 / 2$;

$\%$ The thickness

$\mathrm{t}=13.72$

$\%$ The inner radius

$\mathrm{a}=\mathrm{b}-\mathrm{t}$;

\% Possoin's ratios

$\mathrm{v}=0.3$;

$\mathrm{P} 0=1 / 2 *($ Sig1 + Sig2 $)$;

$\mathrm{b} 2=1 / 2 *(\operatorname{Sig} 1-\operatorname{Sig} 2)$
$\mathrm{A} 2=-\left(\left(\mathrm{a}^{\wedge} 4^{*} \mathrm{~b}^{\wedge} 4 *\left(\mathrm{a}^{\wedge} 2+3 * \mathrm{~b}^{\wedge} 2\right) * \mathrm{~b} 2\right) /\left(6^{*}\left(\mathrm{a}^{\wedge} 2-\right.\right.\right.$ $\left.\left.\left.b^{\wedge} 2\right)^{\wedge} 3\right)\right)$;

$\mathrm{B} 2=\left(\mathrm{a}^{\wedge} 2 * \mathrm{~b}^{\wedge} 2^{*}\left(\mathrm{a}^{\wedge} 4+\mathrm{a}^{\wedge} 2 * \mathrm{~b}^{\wedge} 2+2 * \mathrm{~b}^{\wedge} 4\right) * \mathrm{~b} 2\right) /$ $\left(2 *\left(a^{\wedge} 2-b^{\wedge} 2\right)^{\wedge} 3\right)$;

$\mathrm{C} 2=-\left(\left(\mathrm{b}^{\wedge} 2 *\left(3^{*} \mathrm{a}^{\wedge} 2+\mathrm{b}^{\wedge} 2\right) * \mathrm{~b} 2\right) /\left(6^{*}\left(-\mathrm{a}^{\wedge} 2+\right.\right.\right.$ $\left.\left.b^{\wedge} 2\right)^{\wedge} 3\right)$;

$\mathrm{D} 2=\left(\mathrm{b}^{\wedge} 2 *\left(2 * \mathrm{a}^{\wedge} 4+\mathrm{a}^{\wedge} 2 * \mathrm{~b}^{\wedge} 2+\mathrm{b}^{\wedge} 4\right) * \mathrm{~b} 2\right) /(2 *(-$ $\left.\left.a^{\wedge} 2+b^{\wedge} 2\right)^{\wedge} 3\right)$;

$\mathrm{n}=100 ;$

$\mathrm{r}=$ linspace $(\mathrm{a}, \mathrm{b}, \mathrm{n})$;

$r=r^{\prime}$;

theta $=\mathrm{pi}^{*}(-\mathrm{n}: \mathrm{n}) / \mathrm{n}$

$\mathrm{X}=\mathrm{r} * \cos ($ theta $+\mathrm{pi} / 2)$;

$\mathrm{Y}=\mathrm{r}^{*} \sin ($ theta $+\mathrm{pi} / 2)$

$\operatorname{sigr}=\mathrm{zeros}(\operatorname{size}(\mathrm{Y}))$;

$\operatorname{sigt=}=\operatorname{zeros}(\operatorname{size}(\mathrm{Y}))$;

sigr2=zeros $(\operatorname{size}(Y))$;

$\operatorname{sigt} 2=$ zeros $(\operatorname{size}(\mathrm{Y}))$

tor2=zeros $(\operatorname{size}(\mathrm{Y}))$;

for $\mathrm{i}=1$ :length(r)

for $\mathrm{j}=1$ :length(theta)

$\operatorname{sigr}(\mathrm{i}, \mathrm{j})=\mathrm{a}^{\wedge} 2^{*} \mathrm{~b}^{\wedge} 2^{*}(\mathrm{P}-\mathrm{P} 0) /\left(\left(\mathrm{a}^{\wedge} 2-\mathrm{b}^{\wedge} 2\right)^{*} \mathrm{r}(\mathrm{i})^{\wedge} 2\right)-$

$\left(-\mathrm{a}^{\wedge} 2 * \mathrm{P}+\mathrm{b}^{\wedge} 2 * \mathrm{P} 0\right) /\left(-\mathrm{a}^{\wedge} 2+\mathrm{b}^{\wedge} 2\right)$; 
$\operatorname{sigt}(\mathrm{i}, \mathrm{j})=\left(\mathrm{a}^{\wedge} 2 * \mathrm{P}-\mathrm{b}^{\wedge} 2 * \mathrm{P} 0\right) /\left(-\mathrm{a}^{\wedge} 2+\mathrm{b}^{\wedge} 2\right)-$

$\mathrm{a}^{\wedge} 2 * \mathrm{~b}^{\wedge} 2 *(\mathrm{P}-\mathrm{P} 0) /\left(\left(\mathrm{a}^{\wedge} 2-\mathrm{b}^{\wedge} 2\right)^{*} \mathrm{r}(\mathrm{i})^{\wedge} 2\right)$;

$\operatorname{sigr} 2(\mathrm{i}, \mathrm{j})=-\left(\left(2 *\left(3 * \mathrm{~A} 2+2 * \mathrm{~B} 2 * \mathrm{r}(\mathrm{i})^{\wedge} 2+\right.\right.\right.$

$\left.\left.\left.\mathrm{D} 2 * \mathrm{r}(\mathrm{i})^{\wedge} 4\right)^{*} \cos (2 * \operatorname{theta}(\mathrm{j}))\right) / \mathrm{r}(\mathrm{i})^{\wedge} 4\right)$;

$\operatorname{sigt} 2(\mathrm{i}, \mathrm{j})=\left(2 * \mathrm{D} 2+\left(6^{*} \mathrm{~A} 2\right) / \mathrm{r}(\mathrm{i})^{\wedge} 4+\right.$

$\left.12 * \mathrm{C} 2 * \mathrm{r}(\mathrm{i})^{\wedge} 2\right) * \cos (2 *$ theta $(\mathrm{j}))$;

tor $2(\mathrm{i}, \mathrm{j})=2 * \mathrm{r}(\mathrm{i})^{\wedge}(-4)^{*} \sin (2 *$ theta $(\mathrm{j})) *(-3 * \mathrm{~A} 2-$

$\left.\mathrm{B} 2 * \mathrm{r}(\mathrm{i})^{\wedge} 2+\mathrm{r}(\mathrm{i})^{\wedge} 4 *\left(\mathrm{D} 2+\mathrm{C} 2 *(1+2) * \mathrm{r}(\mathrm{i})^{\wedge} 2\right)\right)$;

end

end

sigr $=$ sigr + sigr 2

sigt=sigt + sigt 2 ;

$\operatorname{sig} \mathrm{z}=\mathrm{v}^{*}(\operatorname{sigr}+\operatorname{sig} \mathrm{t})$

Mises $=\operatorname{sqrt}\left(1 / 2 *\left((\right.\right.$ sigr-sigt $) .^{\wedge} 2+($ sigr-

$\operatorname{sigz}) .^{\wedge} 2+\left(\right.$ sigt-sigz). ${ }^{\wedge} 2+6 *$ tor $\left.\left.2 .^{\wedge} 2\right)\right)$;

figure

pcolor(X,Y,Mises)

shading interp

colorbar

axis equal tight

$\operatorname{caxis}([0,500])$

Maxmises $=\max (\max ($ Mises $))$ 


\title{
حل مغلق الشكل لتحليل الإجهاد لهيكل اسطوانة مجوفة تحت قوة تحميل خارجي غير منتظم وتطبيقاته الهندسية
}

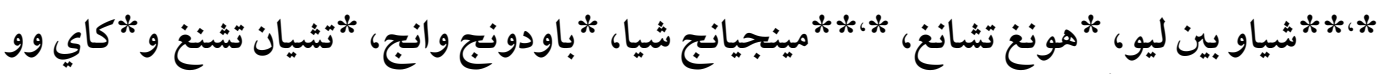

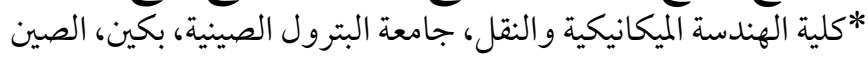

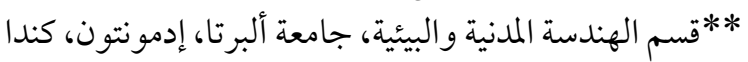

\section{الخالاصة}

تُستخدم الهياكل المجوفة على نطاق واسع في الصناعات المختلفة لقدرتها العالية على التحمل. في بعض الحالات الهندات فيسة،

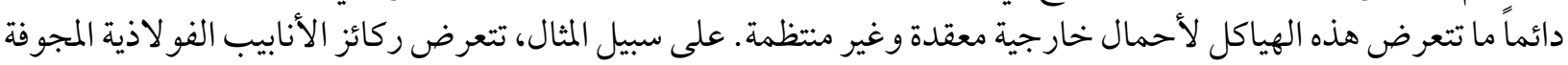

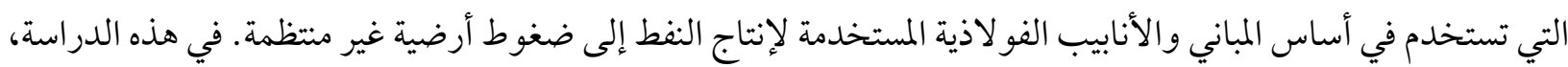

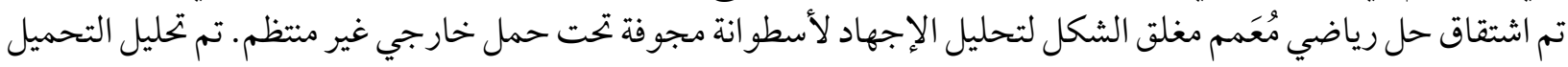

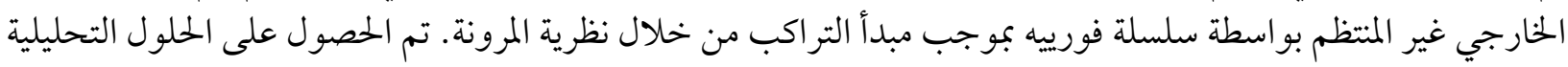
لنتائج الإجهاد لمشاكل التحميل الخارجي لسلسلة الجيب أو سلسلة جيب التمام بو اسطة الطريقة شبه العكسية. تم تقديم تحليل

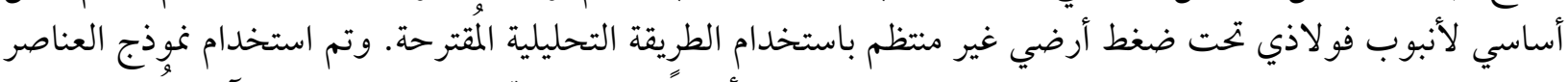

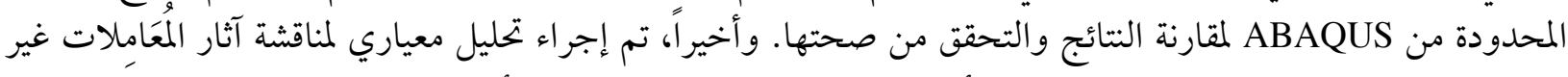

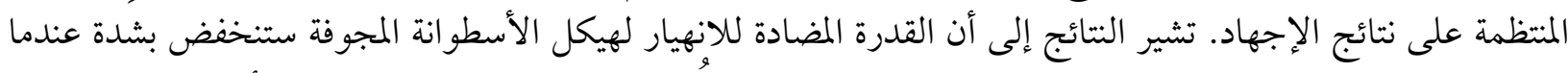

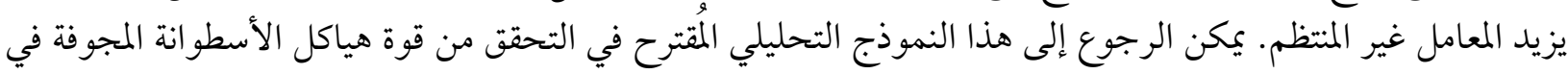

